\title{
A new Size Biased Distribution with Applications in Engineering and Medical Science
}

\author{
${ }^{1 *}$ A. A. Rather, ${ }^{2}$ C. Subramanian, ${ }^{3}$ S Shafi, ${ }^{4}$ K A Malik, ${ }^{5}$ P. J. Ahmad, ${ }^{6}$ B A Para and ${ }^{7}$ T R Jan \\ ${ }^{1,2}$ Department of Statistics, Annamalai University, Chidambaram, Tamil Nadu, India. \\ ${ }^{3}$ Department of Engineering, Islamic University of Science and technology, Awantipora, J\&K, India \\ ${ }^{4}$ Department of Statistics, Govt. Degree College Boys Anantnag, J\&K, India \\ ${ }^{5}$ Department of Statistics, Govt. M.V.M, Bhopal, M.P., India \\ ${ }^{6,7}$ Department of Statistics, University of Kashmir, Srinagar, India \\ *Corresponding Author: aafaq7741@gmail.com
}

Available online at: www.isroset.org

Accepted 18/Aug/2018, Online 30/Aug/2018

Abstract-In this paper, we proposed a new probability model called as Size Biased Ailamujia Distribution (SBAD). Structural properties of this new distribution are discussed comprehensively. Parameter estimation along with simulation study is also discussed thoroughly. Finally, the model is examined with real life data sets for significance purpose.

Keywords: Size biased distribution, Reliability, MLE, Simulation.

\section{INTRODUCTION}

Lv et al. (2002) studied a new probability distribution named as Ailamujia distribution for several engineering applications and discussed its various characteristics. Its probability density function and cumulative density function is given respectively as follows:

$$
\begin{aligned}
& f(x ; \lambda)=4 x \lambda^{2} e^{-2 \lambda x}, \quad x>0, \lambda>0 \\
& F(x ; \lambda)=1-(1+2 \lambda x) e^{-2 \lambda x}, \quad x>0, \lambda>0 .
\end{aligned}
$$

Lot of work has been done in this field. Gove (2003) reviewed some of the more recent results on size-biased distributions pertaining to parameter estimation in forestry. Warren (1975) was the first to apply the size biased distributions in connection with sampling wood cells. More recently; these distributions were used to recover the distribution of canopy heights from airborne laser scanner measurements. Das and Roy (2011) discussed the length-biased Weighted Generalized Rayleigh distribution with its properties. Patil and Ord (1976) introduced the concept of size-biased sampling and weighted distributions by identifying some of the situations where the underlying models retain their form. Ayesha, (2017) discussed the Size Biased Lindley Distribution as a new life time distribution and discussed its various statistical properties. Shankar (2017) discussed a Size-Biased Poisson-Shanker Distribution and its applications to handle various count data sets. Recently, Shanker \& Shukla (2018) discussed a generalized size-biased Poisson-Lindley distribution and Its Applications to model size distribution of freely-forming small group.

\section{SIZE BIASED AILAMUJIA DISTRIBUTION}

If $\mathrm{X}$ is a non negative random variable with probability density function (pdf) $f(x)$, then the probability density function of the size biased random variable $X_{s b}$ is given by:

$$
f_{s b}(x)=\frac{x f(x)}{E(x)}, \quad x>0
$$


The probability density of size biased Ailamujia distribution is given as:

$$
\begin{aligned}
& f_{s b}(x ; \lambda)=\frac{x f(x ; \lambda)}{E(x)}, \\
& f_{s b}(x ; \lambda)=4 \lambda^{3} x^{2} e^{-2 \lambda x}, \quad x>0, \lambda>0,
\end{aligned}
$$

where, $E(x)=4 \lambda^{2} \int_{0}^{\infty} x^{2} e^{-2 \lambda x} d x=\frac{1}{\lambda}$.

The corresponding cdf of size biased Ailamujia distribution is obtained as:

$$
\begin{gathered}
F_{s b}(x ; \lambda)=\int_{0}^{x} 4 \lambda^{3} x^{2} e^{-2 \lambda x} d x \\
F_{s b}(x ; \lambda)=\frac{1}{2} \gamma(3,2 \lambda x), \quad x>0, \lambda>0
\end{gathered}
$$

where $\lambda$ is a positive parameters and $\gamma(s, x)=\int_{0}^{x} z^{s-1} e^{-z} d z$ is a lower incomplete gamma function.

The graphs of probability density function and cumulative distribution function are plotted for different values of parameters $\lambda$ given in Fig. 1and 2 respectively.

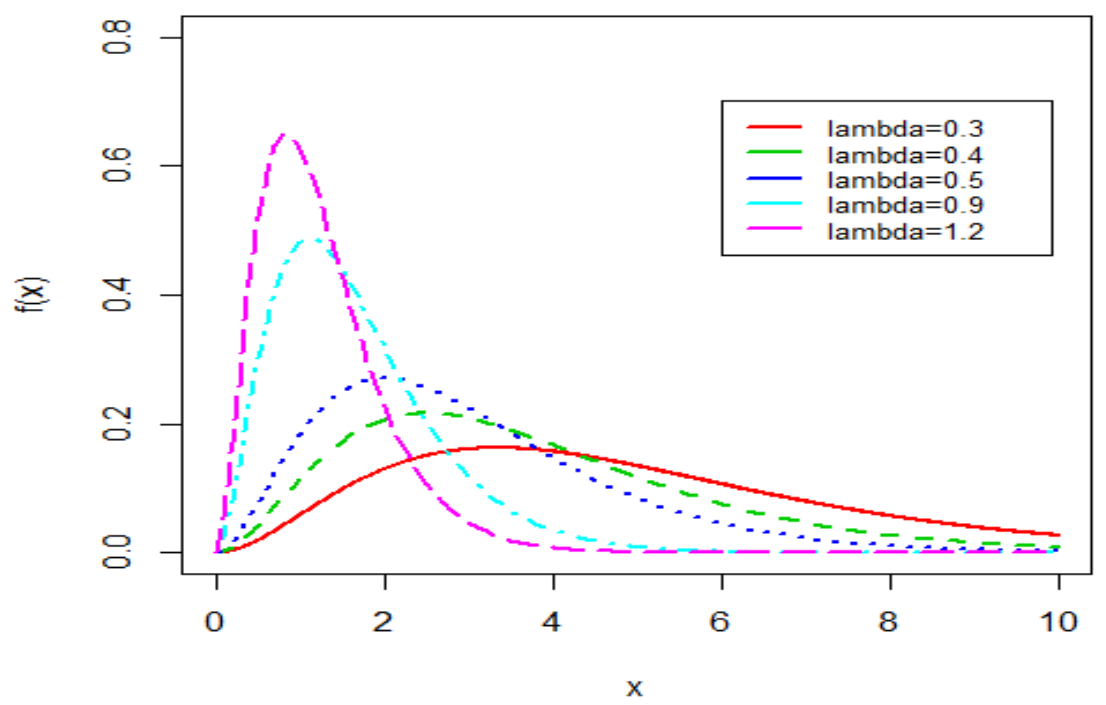

Fig.1: pdf plot of size biased Ailamujia distribution

Fig. 1 gives the description of some of the possible shapes of size biased Ailamujia distribution for different values of the parameter $\lambda$. Fig. 1 illustrates that the density function of size biased Ailamujia distribution is positively skewed. Fig .2 gives the graphical overview of cdf plot of SBAD. As the value of $\lambda$ increases, the initial rise of cdf curve also increases. 


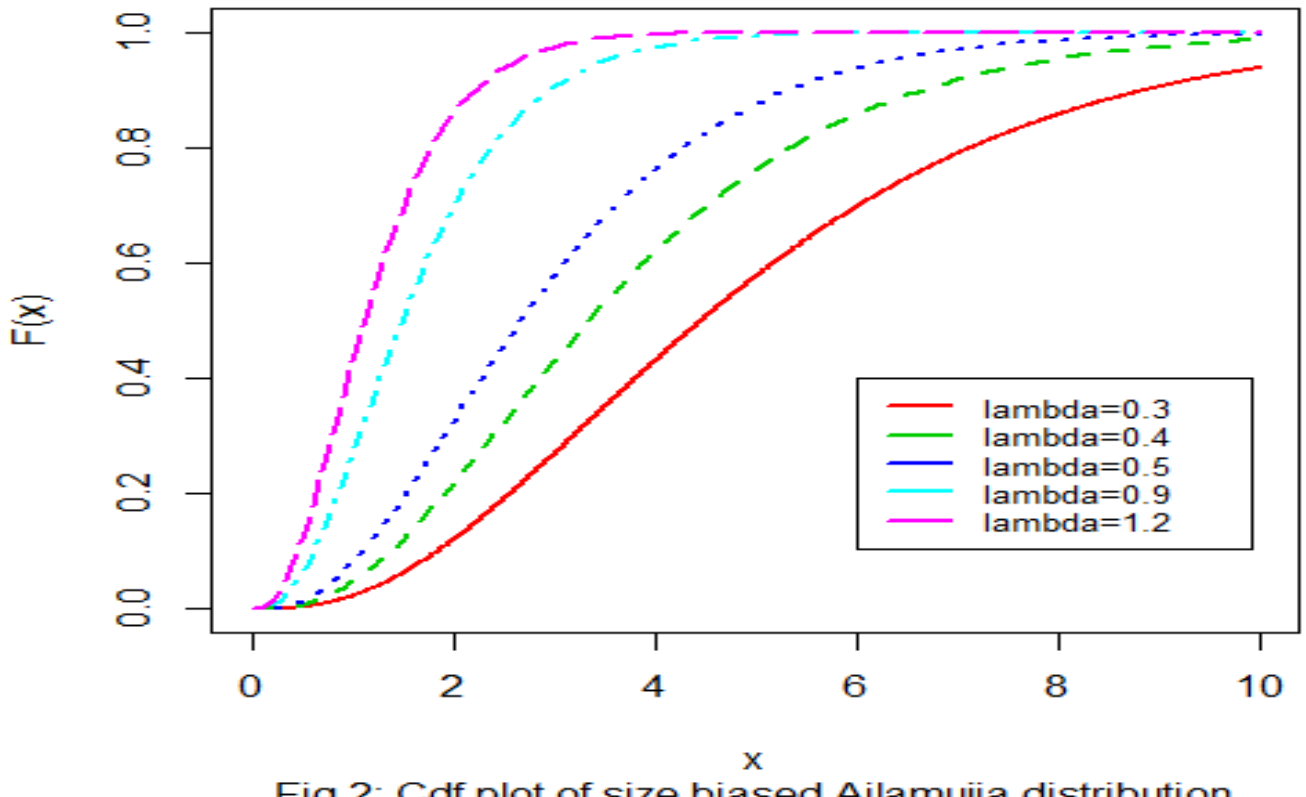

Fig.2: Cdf plot of size biased Ailamujia distribution

\section{QUANTILE AND RANDOM NUMBER GENERATION FROM SBAD}

Inverse cdf method is one of the methods used for the generation of random numbers from a particular distribution. In this method the random numbers from a particular distribution are generated by solving the equation obtained on equating the cdf of a distribution to a number $u$. The number $u$ is itself being generated from uniform distribution i.e., $\mathrm{U}(0,1)$. Thus following the same procedure for the generation of random numbers from the SBAD, we will proceed as

$F_{s b}(x ; \lambda)=u, \quad x>0, \lambda>0$

$F_{s b}(x ; \lambda)=\frac{1}{2} \gamma(3,2 \lambda x)=u, \quad x>0, \lambda>0$

On solving the equation (3.1) for $\mathrm{x}$, we will obtain the required random number from the SBAD.

Equation (3.1) will be solved for $\mathrm{x}$ using R-software.

\section{RELIABILITY ANALYSIS}

In this sub section, we have obtained the reliability, hazard rate, reverse hazard rate and Mills ratio of the proposed size biased Ailamujia model.

4.1 Survival function of $S B A D$

The reliability function is defined as the probability that a system survives beyond a specified time. It is also referred to as survival or survivor function of the distribution. It can be computed as complement of the cumulative distribution function of the model. The reliability function or the survival function of size biased Ailamujia distribution is calculated as:

$$
R_{w}(x, \lambda)=1-\frac{1}{2} \gamma(3,2 \lambda x)(4.1)
$$

The graphical representation of the reliability function for the size biased Ailamujia distribution is shown in fig. 3 


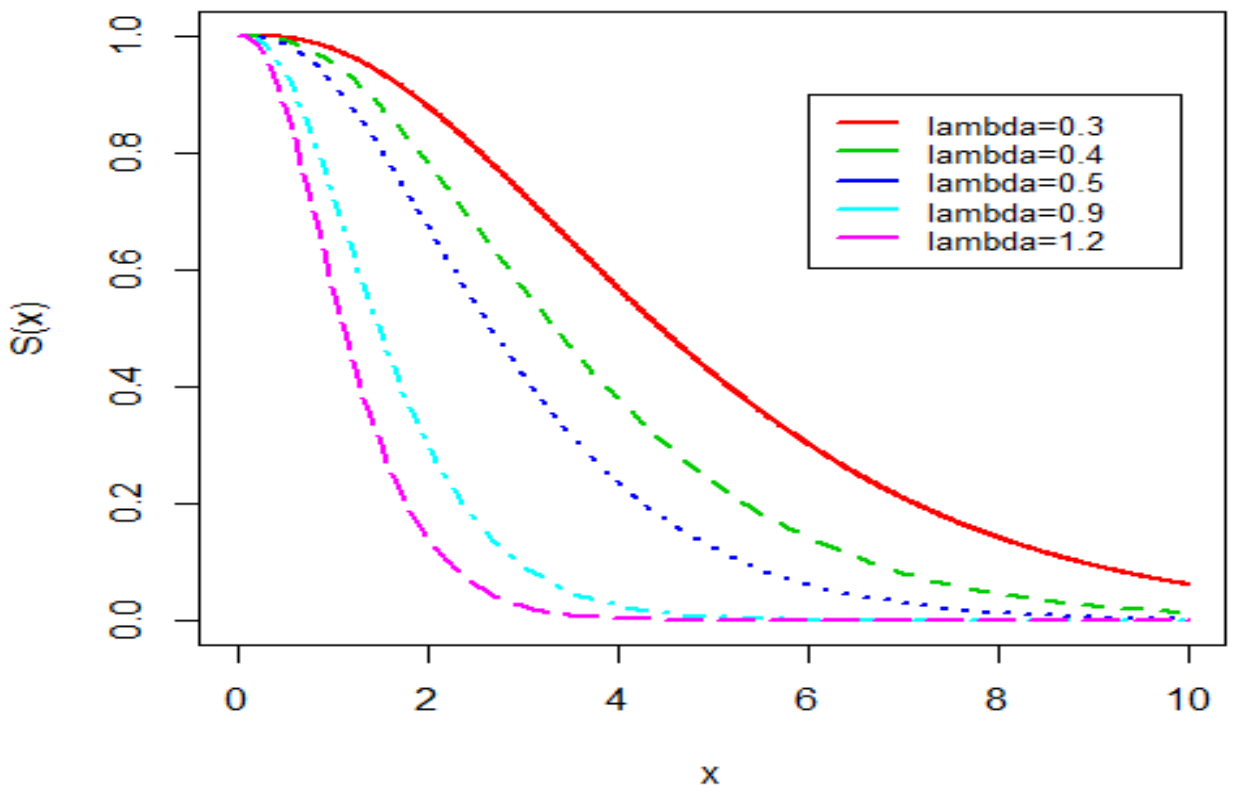

4.2 Hazard Function

Fig.3: Survival function plot of size biased Ailamujia distribution

The hazard function is also known as hazard rate, instantaneous failure rate or force of mortality is given as:

$$
H . R=h(x ; \lambda)=\frac{f_{s b}(x ; \lambda)}{R_{s b}(x ; \lambda)}=\frac{(2 \lambda)^{3} x^{2} e^{-2 \lambda x}}{\Gamma(3,2 \lambda x)} .
$$

\subsection{Reverse Hazard Rate and Mills Ratio}

The reverse hazard rate and the Mills ratio of the size biased Ailamujia distribution are respectively given as:

$$
\begin{aligned}
& \text { R.H.R }=h_{r}(x ; \lambda)=\frac{f_{s b}(x ; \lambda)}{F_{s b}(x ; \lambda)}=\frac{(2 \lambda)^{3} x^{2} e^{-2 \lambda x}}{\gamma(3,2 \lambda x)} . \\
& \text { Mills ratio } \left.=\frac{1}{h_{r}(x ; \lambda)}=\frac{\gamma(3,2 \lambda x)}{(2 \lambda)^{3} x^{2} e^{-2 \lambda x} .} .4 .4\right)
\end{aligned}
$$

\section{STATISTICAL PROPERTIES}

In this section, the different structural properties of the proposed size biased Ailamujia model have been evaluated. These include moments, mode, harmonic mean, moment generating function and characteristic function

5.1 Moments

Suppose $\mathrm{X}$ is a random variable following size biased Ailamujia distribution with parameter $\theta$, and then the rth moment for a given probability distribution is given by

$$
\begin{aligned}
& \mu_{r}^{\prime}=E\left(X_{s b}^{r}\right)=\int_{0}^{\infty} x^{r} f_{s b}(x ; \lambda) d x \\
& =\int_{0}^{\infty} x^{r} 4 \lambda^{3} x^{2} e^{-2 \lambda x} d x \\
& \mu_{r}^{\prime}=\frac{\Gamma(r+3)}{2^{r+1} \lambda^{r}}
\end{aligned}
$$

Substituting $r=1,2,3,4$ we get the first four central moments as follows: 
If we put $\mathrm{r}=1$ in eq. (5.1), we get the mean of size-biased Ailamujia distribution which is given by:

Mean $=\mu_{1}^{\prime}=\frac{3}{2 \lambda}$

If we put $\mathrm{r}=2$ in eq. (5.1), we have

$\mu_{2}^{\prime}=\frac{3}{\lambda^{2}}$.

Thus, the variance of size biased Ailamujia distribution is given as:

Variance $=\mu_{2}=\frac{3}{4 \lambda^{2}}$.

If we put $\mathrm{r}=3$ in eq. (5.1), we have

$$
\mu_{3}^{\prime}=\frac{15}{2 \lambda^{3}} \text {. }
$$

Then $\mu_{3}=\mu_{3}^{\prime}-3 \mu_{2}^{\prime} \mu_{1}^{\prime}+2 \mu_{1}^{\prime 3}$.

Using the values of eq. (5.2), (5.3) and (5.5), we get

$$
\mu_{3}=\frac{3}{4 \lambda^{3}} \text {. }
$$

When we put $r=4$ in eq. (5.1), we get

$$
\mu_{4}^{\prime}=\frac{45}{2 \theta^{4}} \text {. }
$$

Therefore, from the values of eq. (5.2), (5.3), (5.5) and (5.7) we get

$$
\mu_{4}=\frac{45}{16 \lambda^{4}} \text {. }
$$

Standard deviation $=\sigma=\frac{\sqrt{3}}{2 \lambda}$.

Coefficient of variation $=C \cdot V=\frac{\sigma}{\mu}=\frac{1}{\sqrt{3}}$.

5.2 Skewness and Kurtosis of the size-biased Ailamujia distribution

i) Skewness

It may be defined as the lack of symmetry of tails (about mean) of frequency distribution curve. Karl Pearson gave the formula for measuring the skewness of the distribution in terms of the moments of the frequency distribution as:

$$
\beta_{1}=\frac{\mu_{3}{ }^{2}}{\mu_{2}{ }^{3}} \text {. }
$$

After using eq. (5.4) and eq. (5.6) in eq. (5.9), we have

$$
\begin{gathered}
\beta_{1}=\frac{4}{3} . \\
\text { and } \gamma_{1}=\sqrt{\beta_{1}}=\frac{2}{\sqrt{3}} .
\end{gathered}
$$

ii) Kurtosis

It may be defined as the degree of peakedness of the density curve. The formula for obtaining the kurtosis of a distribution in terms of moments is given as:

$$
\beta_{2}=\frac{\mu_{4}}{\mu_{2}^{2}} \text {. }
$$


After using eq. (5.8) and eq. (5.4) in eq. (5.11), we have

$$
\beta_{2}=5
$$

and $\quad \gamma_{2}=\beta_{2}-3=2$.

5.3Mode of size-biased Ailamujia Distribution

In order to calculate the mode of the size biased Ailamujia model we take the logarithm of the probability density function of the size biased model:

$$
\log f_{s b}(x, \lambda)=3 \log 2+3 \log \theta-\log \Gamma(3)+2 \log x-2 \lambda x .
$$

Differentiating the above equation and equating it zero, we obtain the mode as follows:

$$
\begin{gathered}
\frac{d}{d x} \log f_{s b}(x, \lambda)=0 \\
\frac{2}{x}=2 \lambda \\
\Rightarrow \quad x=\frac{1}{\lambda} .
\end{gathered}
$$

\subsection{Harmonic mean}

The harmonic mean for the proposed model is computed as:

$$
\begin{aligned}
H . M & =E\left[\frac{1}{X}\right]=\int_{0}^{\infty} \frac{1}{x} f_{s b}(x ; \lambda) d x \\
& =\int_{0}^{\infty} \frac{1}{x} 4 \lambda^{3} x^{2} e^{-2 \lambda x} d x \\
H . M & =\lambda .
\end{aligned}
$$

5.5Moment generating function and characteristic function

This section deals with the derivation of moment generating function and characteristic function of the size biased Ailamujia distribution. By the definition of moment generating function, we have:

$$
M_{X}(t)=E\left(e^{t x}\right)=\int_{0}^{\infty} e^{t x} f_{w}(x ; \lambda) d x .
$$

Using Taylor series

$$
\begin{aligned}
M_{X}(t) & =\int_{0}^{\infty}\left(1+t x+\frac{(t x)^{2}}{2 !}+\cdots\right) f_{s b}(x ; \lambda) d x \\
\Rightarrow \quad M_{X}(t) & =\sum_{r=0}^{\infty} \frac{t^{k}}{k !} \int_{0}^{\infty} x^{k} f_{s b}(x ; \lambda) d x \\
\Rightarrow \quad M_{X}(t) & =\sum_{k=0}^{\infty} \frac{t^{k}}{k !} E\left(X^{k}\right) \\
\Rightarrow \quad M_{X}(t) & =\sum_{k=0}^{\infty} \frac{\left(\frac{t}{2 \lambda}\right)^{k}}{k !} \frac{\Gamma(k+3)}{\Gamma(3)} .
\end{aligned}
$$

Similarly, the characteristic function of size biased Ailamujia distribution is computed as:

$$
\phi_{X}(t)=E\left(e^{i t x}\right)=\int_{0}^{\infty} e^{i t x} f_{s b}(x ; \lambda) d x .
$$

Using Taylor series 


$$
\begin{aligned}
\phi_{X}(t) & =\int_{0}^{\infty}\left(1+i t x+\frac{(i t x)^{2}}{2 !}+\cdots\right) f_{s b}(x ; \lambda) d x \\
\Rightarrow \quad \phi_{X}(t) & =\sum_{k=0}^{\infty} \frac{(i t)^{k}}{k !} \int_{0}^{\infty} x^{k} f_{s b}(x ; \lambda) d x \\
\Rightarrow \quad \phi_{X}(t) & =\sum_{k=0}^{\infty} \frac{(i t)^{k}}{k !} E\left(X^{k}\right) \\
\Rightarrow \quad \phi_{X}(t) & =\sum_{k=0}^{\infty} \frac{\left(\frac{i t}{2 \lambda}\right)^{k}}{k !} \frac{\Gamma(k+3)}{\Gamma(3)} .
\end{aligned}
$$

\section{SHANNON'S ENTROPY OF SIZE-BIASED AILAMUJIA DISTRIBUTION}

The Shannon entropy of a random variable $\mathrm{X}$ is a measure of the uncertainty and is given by $E[-\log (f(x))]$, where $f(x)$ is the probability function of the random variable $\mathrm{X}$. The Shannon's entropy of the size biased Ailamujia model as follows:

$$
\begin{aligned}
& H(x, \theta)=-E[\log f(x ; \lambda)] \\
& =-E\left[\log \left(4 \lambda^{3} x^{2} e^{-2 \lambda x}\right)\right] \\
& =-\log \left(4 \lambda^{3}\right)-E[2 \log x-2 \lambda x] \\
& H(x, \lambda)=-2 \log 2-3 \log \lambda-2 E[\log x]-2 \lambda E(x) H(x, \lambda)=-2 \log 2-3 \log \lambda-2 I_{1}-2 \lambda E(x) .(6.1)
\end{aligned}
$$

Now, $I_{1}=E(\log x)=\int_{0}^{\infty} \log x f(x) d x$

$$
=4 \lambda^{3} \int_{0}^{\infty} \log (x) x^{2} e^{-2 \lambda x} d x
$$

Put $2 \lambda x=t$, as $x \rightarrow 0, t \rightarrow 0$; as $x \rightarrow \infty, t \rightarrow \infty, d x=\frac{d t}{2 \lambda}$.

On solving (6.2) we have,

$$
I_{1}=\psi(3)-\log 2 \lambda,
$$

Where $\psi($.$) is the digamma function.$

Using the values of (6.3) and (5.1) in (6.1)

$H(x, \lambda)=-2 \log 2-3 \log \lambda-2[\psi(3)-\log 2 \lambda]-3(6.4)$

\section{ORDER STATISTICS}

Let $X_{(1)}, X_{(2)}, X_{(3)} \ldots ., X_{(n)}$ be the ordered statistics of the random sample $X_{1}, X_{2}, X_{3}, \ldots . X_{n}$ drawn from the continuous distribution with cumulative distribution function $F_{X}(x)$ and probability density function $f_{X}(x)$, then the probability density function of rth order statistics $X_{(r)}$ is given by:

$$
f_{x(r)}(x, \theta)=\frac{n !}{(r-1) !(n-r) !} f(x)[F(x)]^{r-1}[1-F(x)]^{n-r} \cdot r=1,2,3, \ldots, \mathrm{n}
$$

Using the equations (2.1) and (2.2), the probability density function of rth order statistics of size biased Ailamujia distribution is given by: 


$$
f_{s b(r)}(x, \theta)=\frac{n !}{(r-1) !(n-r) !} 4 \lambda^{3} x^{2} e^{-2 \lambda x}\left[\frac{1}{2} \gamma(3,2 \lambda x)\right]^{r-1}\left[1-\frac{1}{2} \gamma(3,2 \lambda x)\right]^{n-r} .
$$

Then, the pdf of first order $X_{(1)}$ size biased Ailamujia distribution is given by:

$$
f_{s b(1)}(x, \theta)=n 4 \lambda^{3} x^{2} e^{-2 \lambda x}\left[1-\frac{1}{2} \gamma(3,2 \lambda x)\right]^{n-1} .
$$

and the pdf of nth order $X_{(n)}$ size biased Ailamujia model is given as:

$$
f_{s b(n)}(x, \theta)=n 4 \lambda^{3} x^{2} e^{-2 \lambda x}\left[\frac{1}{2} \gamma(3,2 \lambda x)\right]^{n-1} \text {. }
$$

\section{ESTIMATION OF PARAMETERS}

In this section, we estimate the parameters of the size biased Ailamujia distribution using different methods.

\subsection{Method of Moments}

In order to obtain sample moments, we replace population moments with sample moments:

$$
\begin{aligned}
& \frac{\sum_{i=1}^{n} x_{i}}{n}=\mu_{1}, \\
\Rightarrow \quad & \bar{x}=\frac{3}{2 \theta} \\
\Rightarrow \quad & \hat{\lambda}=\frac{3}{2 \bar{x}}
\end{aligned}
$$

8.2 Method of Maximum Likelihood Estimation

This is one of the most useful method for estimating the different parameters of the distribution. Let $X_{1}, X_{2}, X_{3} \ldots . . X_{n}$ be the random sample of size $\mathrm{n}$ drawn from size biased Ailamujia distribution, then the likelihood function of size biased Ailamujia distribution is given as:

$$
L(x \mid \lambda)=\frac{(2 \lambda)^{3 n}}{[\Gamma(3)]^{n}} \prod_{i=1}^{n} x_{i}^{2} e^{-2 \lambda \sum_{i=1}^{n} x_{i}}
$$

The log likelihood function becomes:

$$
\log L=3 n \log 2+3 n \log \lambda-n \log \Gamma(3)+2 \sum_{i=1}^{n} \log x_{\imath}-2 \lambda \sum_{i=1}^{n} x_{i} .
$$

Differentiating the log-likelihood function with respect to $\lambda$. equating the result to zero, we obtain the following normal equations,

$$
\begin{aligned}
& \frac{d \log L}{d \lambda}=\frac{n(c+2)}{\lambda}-2 \sum_{i=1}^{n} x_{i}=0 . \\
& \Rightarrow \quad \hat{\lambda}=\frac{3}{2 \bar{x}}
\end{aligned}
$$

The maximum likelihood estimator and Moments method coincide for estimation of parameter $\lambda$ of the size biased Ailamujia distribution. 


\subsection{Simulation Study of ML Estimators}

In this section, we study the performance of ML estimators for different sample sizes $(\mathrm{n}=25,50,75,100,150,300)$. We have employed the inverse CDF technique for data simulation for size biased Ailamujia distribution using R software. The process was repeated 500 times for calculation of bias, variance and MSE. For different values of parameters of size biased Ailamujia distribution, decreasing trend is being observed in average bias, variance and MSE as we increase the sample size. Hence, the performance of ML estimators is quite well, consistent in case of size biased Ailamujia distribution.

Table 1: Bias, Variance and MSE for ML estimators for Different Sample Sizes.

\begin{tabular}{|c|c|c|c|c|c|c|}
\hline \multirow{2}{*}{ Sample Size n } & \multicolumn{3}{|c|}{$\lambda=0.3$} & \multicolumn{3}{|c|}{$\lambda=0.5$} \\
\hline & Bias & Variance & MSE & Bias & Variance & MSE \\
\hline & 0.0022910 & 0.0010869 & 0.0010922 & 0.0078600 & 0.0029535 & 0.0030152 \\
\hline 50 & 0.0011809 & 0.0006388 & 0.0006402 & 0.0014040 & 0.0017889 & 0.0017908 \\
\hline 75 & -0.0016428 & 0.0004165 & 0.0004192 & 0.0032969 & 0.0011023 & 0.0011132 \\
\hline 100 & 0.0030825 & 0.0003127 & 0.0003222 & 0.0023543 & 0.0009113 & 0.0009169 \\
\hline 150 & 0.0005556 & 0.0001963 & 0.0001966 & -0.0003754 & 0.0006998 & 0.0007000 \\
\hline \multirow[t]{3}{*}{300} & 0.0007411 & 0.0000965 & 0.0000971 & 0.0033082 & 0.0003294 & 0.0003404 \\
\hline & \multicolumn{3}{|c|}{$\lambda=1.5$} & \multicolumn{3}{|c|}{$\lambda=1.8$} \\
\hline & Bias & Variance & MSE & Bias & Variance & MSE \\
\hline 25 & 0.0241798 & 0.0422345 & 0.0428191 & 0.0654938 & 0.0496775 & 0.0539669 \\
\hline 50 & 0.0169685 & 0.0165570 & 0.0168449 & 0.0305102 & 0.0260309 & 0.0269618 \\
\hline 75 & 0.0767764 & 0.0062306 & 0.0121252 & 0.0011220 & 0.0154117 & 0.0154130 \\
\hline 100 & 0.0145912 & 0.0062579 & 0.0064708 & 0.0129767 & 0.0102880 & 0.0104564 \\
\hline 150 & 0.0024394 & 0.0047430 & 0.0047490 & 0.0104862 & 0.0073863 & 0.0074963 \\
\hline \multirow[t]{3}{*}{300} & 0.0181557 & 0.0019860 & 0.0023156 & 0.0108284 & 0.0033157 & 0.0034330 \\
\hline & \multicolumn{3}{|c|}{$\lambda=2.6$} & \multicolumn{3}{|c|}{$\lambda=3.5$} \\
\hline & Bias & Variance & MSE & Bias & Variance & MSE \\
\hline 25 & 0.0379822 & 0.1016088 & 0.1030515 & 0.0191421 & 0.1505014 & 0.1508678 \\
\hline 50 & 0.0483251 & 0.0552168 & 0.0575522 & 0.0296694 & 0.0996841 & 0.1005644 \\
\hline 75 & -0.0161357 & 0.0218196 & 0.0220800 & 0.0725376 & 0.0352564 & 0.040518 \\
\hline 100 & 0.0375621 & 0.0192373 & 0.0206482 & -0.1005993 & 0.0284668 & 0.0385870 \\
\hline 150 & 0.0105398 & 0.0181228 & 0.0182339 & 0.0338643 & 0.0262681 & 0.0274149 \\
\hline 300 & -0.0173914 & 0.0065915 & 0.0068940 & 0.0087072 & 0.0102951 & 0.0103710 \\
\hline
\end{tabular}

\section{APPLICATIONS OF SIZE-BIASED AILAMUJIA DISTRIBUTIONS}

In this section, we present the goodness of fit of Size biased Ailamujia distribution using maximum likelihood estimate of the parameter on two data sets and the fit has been compared with Ailamujia distribution. For testing the goodness of fit of Size biased Ailamujia distribution over Ailamujia distribution, following two data sets have been considered.

(i) Data given in table 2, arose in tests on endurance of deep groove ball bearings. The data are the number of million revolutions before failure for each of the 23 ball bearings in the life tests.

Table 2: Number of million revolutions before failure for each of the 23 ball bearings in the life tests studied by Lawless [2]

\begin{tabular}{|r|r|r|r|r|r|r|r|}
\hline 17.88 & 28.92 & 33 & 41.52 & 42.12 & 45.6 & 48.8 & 51.84 \\
\hline 51.96 & 54.12 & 55.56 & 67.8 & 68.44 & 68.64 & 68.88 & 84.12 \\
\hline 93.12 & 98.64 & 105.12 & 105.84 & 127.92 & 128.04 & 173.4 & \\
\hline
\end{tabular}


In order to compare the two models, we consider the AIC (Akaike information criterion), AICC (corrected Akaike information criterion) and BIC (Bayesian information criterion) criterion. The better distribution corresponds to lesser AIC, AICC and BIC values. The generic formulas for finding AIC, AICC and BIC are given as

$$
\mathrm{AIC}=2 \mathrm{k}-2 \log L \quad \mathrm{AICC}=\mathrm{AIC}+\frac{2 k(k+1)}{n-k-1} \text { and } \mathrm{BIC}=\mathrm{k} \log n-2 \log ,
$$

where $\mathrm{k}$ is the number of parameters in the statistical model, $\mathrm{n}$ is the sample size and $-\log L$ is the maximized value of the $\log$ likelihood function under the considered model. From Table 3, it has been observed that size biased Ailamujia distribution have the lesser AIC, AICC, $-\log L$ and BIC values as compared to Ailamujia Distribution. Hence we can conclude that the size biased Ailamujia distribution leads to a better fit than the Ailamujia distribution. Kolmogorov Smirnov p-value suggests that size biased Ailamujia distribution fits statistically better than Ailamujia distribution to a data set given in table 2 .

Table 3: Model Comparison using AIC, AICC, BIC, -logL Criterion and Likelihood Ratio Test for data given in table 1, reported by Lawless[2]

\begin{tabular}{|c|c|c|c|c|c|c|}
\hline Criteria & $-\operatorname{logL}$ & AIC & AICC & BIC & $\begin{array}{c}\text { KS- } \\
\text { Distance }\end{array}$ & KS P-value \\
\hline Size Biased Ailamujia Distribution & 113.5195 & 229.0391 & 229.2295 & 230.1745 & 0.1190 & 0.8627 \\
\hline Ailamujia Distribution & 115.5242 & 233.0484 & 233.2389 & 234.1839 & 0.1887 & 0.3422 \\
\hline
\end{tabular}

(ii) The following data represent 40 patients suffering from blood cancer (leukemia) from one of Ministry of Health Hospitals in Saudi Arabia (see Abouammahet al.). The ordered lifetimes (in years) are given in table 3.

(iii)

Table 4: Lifetimes (in days) of 40 patients suffering from blood cancer (leukemia) from one of Ministry of Health Hospitals in Saudi Arabia.

\begin{tabular}{|r|r|r|r|r|r|r|r|r|}
\hline 0.315 & 0.496 & 0.616 & 1.145 & 1.208 & 1.263 & 1.414 & 2.025 & 2.036 \\
\hline 2.162 & 2.211 & 2.37 & 2.532 & 2.693 & 2.805 & 2.91 & 2.912 & 3.192 \\
\hline 3.263 & 3.348 & 3.348 & 3.427 & 3.499 & 3.534 & 3.767 & 3.751 & 3.858 \\
\hline 3.986 & 4.049 & 4.244 & 4.323 & 4.381 & 4.392 & 4.397 & 4.647 & 4.753 \\
\hline 4.929 & 4.973 & 5.074 & 5.381 & & & & & \\
\hline
\end{tabular}

Model comparison criterion like AIC, AICC, BIC, -logL suggest that size biased Ailamujia distribution leads to a better fit than the Ailamujia distribution (See table 4). Also it is clear that Kolmogorov Smirnov p-value is statistically significant in case of size biased Ailamujia distribution where as Kolmogorov Smirnov p-value is statistically not significant in case of Ailamujia distribution. Hence, we conclude that data given in table 4 follows size biased Ailamujia distribution and is appropriate for analyzing this data.

Table 4: Model Comparison using AIC, AICC, BIC, - $\operatorname{logL}$ Criterion and KS Distance and KS p-values for data given in table 3.

\begin{tabular}{|c|c|c|c|c|c|}
\hline Criterion & $-\operatorname{logL}$ & AIC & AICC & BIC & KS-Distance P- \\
\hline $\begin{array}{c}\text { Size Biased Ailamujia } \\
\text { Distribution }\end{array}$ & 73.766 & 149.53 & 149.63 & 151.22 & 0.16269 \\
\hline & 76.374 & 154.74 & 154.85 & 0.2402 \\
Ailamujia Distribution & & & & 0.09709 \\
\hline
\end{tabular}

\section{CONCLUSION}

This manuscript deals with the size-biased Ailamujia distribution and studies its different statistical properties. In this paper, moments, mode, harmonic mean, survival function and hazard rate, method of moments and maximum likelihood estimates of the parameters have been obtained. The newly proposed model has been applied to the different real life data sets and the results obtained prove that the proposed size biased Ailamujia model is better fit than its sub models. 


\section{REFERENCES}

[1]. Abouammoh, A.M., Ahmed, R. and Khalique, A. (2000). On new renewal better than used classes of life distribution. Statistics and Probability Letters, 48, 189-194.

[2]. Ayesha, A. (2017). Size Biased Lindley Distribution and Its Properties a Special Case of Weighted Distribution. Applied Mathematics, 08(06), 808-819.doi:10.4236/am.2017.86063

[3]. Das, K. K., and Roy, T. B. (2011). Applicability of Length Biased Weighted Generalized Rayleigh Distribution, Advances in Applied Science Research, 2011, 2 (4), 320-327.

[4]. Gove, J. H. (2003). Environmental and Ecological Statistics, 10(4), 455-467. doi:10.1023/a:1026000505636

[5]. Lawless JF (2003) Statistical models and methods for lifetime data. John Wiley and Sons, New York, USA.

[6]. Lv H. Q., Gao L. H., Chen C. L. (2002). Эрланга distribution and its application in supportability data analysis. Journal of Academy of Armored Force Engineering, 16(3): 48-52.

[7]. Patil, G.P. and Ord, J.K. (1976) On size-biased sampling and related form-invariant weighted distributions. Sankhya, Series B, 38(1), 4861.

[8]. Rama Shankar (2017), A Size-Biased Poisson-Shanker Distribution and Its Applications. International Journal of Probability and Statistics, 6(3):33-44

[9]. Shanker, R., \&Shukla, K. K. (2018). A Generalized Size-Biased Poisson-Lindley Distribution and Its Applications to Model Size Distribution of Freely-Forming Small Group. Journal of Scientific Research, 10(2), 145. doi:10.3329/jsr.v10i2.34905

[10]. Warren, W. G. (1975). Statistical Distributions in Forestry and Forest Products Research. A Modern Course on Statistical Distributions in Scientific Work, 369-384. doi:10.1007/978-94-010-1845-6_27 